

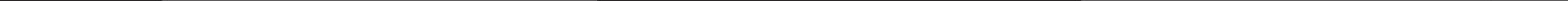




\section{Una aproximación a territorios en situación de conflicto armado: perspectivas desde la Economía Solidaria con un enfoque triangulado en dos municipios del Cauca (Colombia)}

Por Malen Yudeli Solís Montenegro*, Miguel Andrés Ibarra Bermúdez**, Fabián Enrique Salazar Villano***

Resumen: el auge de los procesos de negociación en Colombia entre el Gobierno y grupos insurgentes ha generado un amplio panorama tanto de investigación académica como de acción pública, que conlleva a la reflexión sobre las condiciones necesarias para la construcción de paz territorial en las zonas rurales del país. En tal dirección, en este documento se propone una lectura del conflicto armado desde los fundamentos y prácticas de la Economía Solidaria, y la utilización de una metodología triangulada o mixta, que incorpore las voces de los actores locales con el fin de identificar iniciativas sociales construidas de "Abajo-hacia-Arriba", para así configurar nuevas agendas temáticas en zonas rurales como las del departamento del Cauca en Colombia, tal como se realiza aquí para dos municipios de referencia.

Palabras clave: Conflicto armado, economía solidaria, territorio, sector rural.

* Economista Universidad del Cauca. Investigadora del Semillero "Economía Social y Cultura de paz”, Grupo Pensamiento económico, Sociedad y Cultura de la Universidad del Cauca. Contacto: mysolis@unicauca.edu.co

** Economista Universidad del Cauca. Investigador del Semillero "Economía Social y Cultura de paz”, Grupo Pensamiento económico, Sociedad y Cultura de la Universidad del Cauca. Contacto: maibarra@unicauca.edu.co

*** Economista, Especialista en Gerencia de Proyectos, Magister en Gestión de Organizaciones. Investigador del Semillero “Economía Social y Cultura de paz”, adscrito al Grupo Pensamiento económico, Sociedad y Cultura de la Universidad del Cauca. Contacto: fesalazar@unicauca.edu.co 


\section{An approach to territories with armed conflict condition: perspectives since Solidarity Economics and triangulated approach in two municipalities of Cauca (Colombia)}

Abstract: The height of negotiation processes in Colombia between the Government and insurgent groups has generated a broad outlook for academic research as well as public intervention, which lead to reflect on the needed conditions for territorial peace construction in the rural areas of the country. In this regard, the document proposes an interpretation for the armed conflict since the foundations and practices of Solidarity Economics. In addition, a triangulated methodology is applied (that incorporates voices of local actors in order to identify "Bottom-up" social initiatives), in order to set up new thematic agendas in rural areas such as those of the department of Cauca in Colombia, in the way as it is done here for two reference municipalities.

Keywords: Armed Conflict, Solidarity Economics, Territory, Rural Sector.

Cómo citar este artículo: Solís, Malen; Ibarra, Miguel y Salazar, Fabián (2018). Una aproximación a territorios en situación de conflicto armado: perspectivas desde la Economía Solidaria con un enfoque triangulado en dos municipios del Cauca (Colombia). Revista Controversia, 210, 179-200.

Fecha de recepción: 19 de enero del 2018

Fecha de aprobación: 9 de abril del 2018

\section{Introducción}

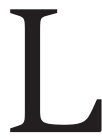

os procesos de negociación de paz en Colombia entre el Gobierno y los grupos insurgentes han descubierto un panorama de investigación y de acción pública que suscita el debate en torno a las condiciones para la construcción de paz territorial con las comunidades rurales, sobretodo en el nivel socio-económico. En esa dirección, el presente artículo realiza un rastreo al conflicto en la teoría económica, que diferencia la forma como se ha tratado esta variable en las vertientes ortodoxas, y en algunas visiones alternativas, en la medida que el primer enfoque parte de supuestos ideales conducentes al bienestar social, alejándose de una serie de condiciones estructurales explicativas del conflicto, como son las pugnas, las desigualdades sociales y las instituciones (entendidas como patrones culturales de 
comportamiento). En ese sentido, también se plantean las prácticas económicas solidarias, y su enfoque territorial en el caso rural, como alternativas para la superación del conflicto. En segundo lugar, el documento argumenta que una visión económica solidaria y territorial para el estudio del conflicto implica el uso de herramientas metodológicas distintas, de aquí que se exponga la metodología triangulada, que combina teoría, actores del territorio y valoraciones de los investigadores en contexto, para aproximarse a entornos rurales en conflicto y derivar acciones de transformación territorial futura. Finalmente, se efectúa una aplicación de estas ideas en el contexto de dos municipios del departamento del Cauca altamente golpeados por el conflicto armado (El Tambo y Patía), de los cuales se concluye la pertinencia de la aproximación propuesta en otras zonas del país.

\section{Revisión teórica}

Desde sus inicios, la literatura económica ha hecho omisión del conflicto como un hecho trascendental para las dinámicas de las sociedades; desde Smith, la teoría económica se torna aislada de los conflictos ya que, por definición, se asume que cada sujeto conoce toda la información disponible, y por ello tiene la capacidad de analizarla y evaluarla, al punto que tiende a maximizar su utilidad y minimizar sus riesgos (Rawls, 2006), es decir prefiriendo más de "lo bueno" y menos de "lo que le cause mal", operando por lo tanto en un contexto donde no existe conflicto de visiones ni intereses. Dicha tendencia epistemológica trasciende con la perspectiva neoclásica plasmada en los trabajos de Haavelmo (1944), y más adelante Becker (1968), quienes plantean modelos de "comportamiento racional"; este último, por ejemplo, propone que los agentes dividen sus actividades en dos tipologías: unas legales y otra ilegales, y que por tanto, un individuo racional se vuelve criminal cuando los beneficios monetarios y de otro tipo obtenidos por actividades ilegales son superiores a los obtenidos por su trabajo de manera legal. En resumen, la perspectiva ortodoxa, con sus formulaciones inductivas y positivistas, presenta argumentos extremadamente limitados 
para la comprensión integral del conflicto social; por lo tanto es necesario explorar aquellas visiones heterodoxas que han tenido un mayor acercamiento al contexto de las sociedades en pugna, puesto que hacen evidentes las falencias del modelo económico convencional fundamentado en el carácter de homo economicus o de elección racional (donde importan solo la maximización de ingresos y la reducción de costos).

Entre estas teorías heterodoxas se encuentra la Economía Política de Marx (2004), que visibilizó los conflictos de su época, manifiestos en las profundas brechas entre proletariado y burguesía, y la dominación y explotación (extracción de plusvalía) ejercida por esta última clase sobre la primera; igualmente, se pueden situar aquí los argumentos de la Escuela Institucionalista, que argumenta la interdependencia de las acciones de cada persona respecto del bienestar de los demás (Bowles, 2010), y el claro papel de la jerarquización de la sociedad como causa de conflictos (González, 1995; Veblen, 2000); por último, se puede encontrar una lectura diferente del conflicto en la aproximación para América Latina de la Escuela Estructuralista con Prebisch (1983) y el modelo Centro-Periferia, esquema analítico según el cual existen centros formados por economías fuertemente diversificadas, con alto grado de progreso técnico, y por ende productividad, mientras las periferias se caracterizan por su rezago tecnológico, reducida diversificación económica, y gran heterogeneidad de productividad interna, modelo que en escala puede ser adaptado para estudiar las brechas entre entornos rurales y urbanos.

Hasta aquí, se han situado en términos generales algunos los aportes desde los cuerpos teóricos más significativos de la Economía como disciplina en torno a la ausencia o existencia de conflicto. No obstante, para hacer una lectura más pertinente del conflicto en entornos de ruralidad es necesario atender a renovados enfoques, y para ello, recurrir a constructos teóricos como la Economía Social y Solidaria, la cual resalta el papel de economías alternativas construidas de "Abajo-hacia-Arriba”, para así contribuir a nuevas agendas temáticas en sectores como 
el rural. Lo anterior, entre otras cosas, porque los modelos de desarrollo capitalista han visto al sector primario como aquel referente de rezago, pobreza, inferioridad, improductividad, sin descubrir sus potencialidades (Salgado, 2005), así que resulta “crucial avanzar en la construcción de otra economía que cambie las reglas del sistema global vigente [... es decir se trata de gestar] otra economía, otro mundo. Esta es la Economía Popular y Solidaria” (Jácome, et. al., 2016, p. 19-20).

Para una mejor comprensión de las economías alternativas es importante considerar a Coraggio, quien explica el concepto de Economía Social y Solidaria así: “i) Es un sistema económico cuyo funcionamiento asegura la base material integrada a una sociedad justa y equilibrada; ii) es un proyecto de acción colectiva (incluyendo prácticas estratégicas de transformación y cotidianas de reproducción) dirigido a contrarrestar las tendencias socialmente negativas del sistema existente, con la perspectiva (actual o potencial) de construir un sistema económico alternativo" (Coraggio, 2011, p. 380). En tal forma, se coloca al factor humano y su trabajo en el centro del sistema económico, y no al mercado y al dinero como convencionalmente sucede, llamando la atención sobre la importancia del diálogo y la cooperación, el reconocimiento de las necesidades comunitarias, y el diseño de estrategias para su gestión colectiva.

En esa dirección se puede afirmar que la Economía de la solidaridad es uno de los mecanismos para atenuar los conflictos sociales, al dar prevalencia a la confianza comunitaria y a la construcción de tejido social en lo rural (Schejiman, 1980), dejando de lado un elemento fundamental de la economía convencional: el egoísmo. Esta idea es defendida por Pastore (2006), quien define la Economía Solidaria en términos de una re-conceptualización de la Economía tradicional, como una respuesta asociativa diferenciada frente a la problemática de exclusión propia del contexto globalizado; en ello coincide con el pensamiento de Razeto (1995), quien explica el concepto del "Factor C", un elemento intangible que conjuga todas aquellas nuevas formas de relacionamiento social basadas en la cooperación (Agudelo, Patiño y Ramos, 2010, con 
base en Razeto (1995)). Como lo explica Primavera, se trata entonces de "un modelo de economía destinado a producir bienestar colectivo y no acumulación de riqueza en pocas manos” (Primavera, 2011, p.7).

Así las cosas, mientras las miradas ortodoxas niegan el conflicto o le quitan un lugar importante en el análisis económico y social convencional, el pensamiento alternativo (y dentro este se incluye la Economía Solidaria) se decide por razonamientos distintos, los cuales tienen por categoría analítica fundamental el reconocimiento de las divergencias sociales y la diversidad socio-cultural, siendo para ello importante comprender el territorio, más allá de su delimitación política y uso productivo (Moncayo, 2001; Moncayo 2003), para centrarse en las relaciones humanas.

Como se ha querido destacar hasta aquí, un análisis económico solidario del conflicto implica tener una noción amplia de lo que se entiende por territorio: para Manzano (2005), el territorio es un escenario social de complejidad útil para entender las pugnas, pero también en contraste, para analizar el surgimiento de formas comunitarias integradoras emanadas de la diversidad productiva y social. Como bien lo expresan Pérez y Uribe, el territorio es una “construcción social, política, económica, geográfica y espacial, donde se reconoce la presencia de diversos actores, los cuales definen relaciones sociales, culturales e institucionales, que se apropian de la espacialidad dándole significado, es decir, el espacio es percibido, concebido y construido con la sinergia de todos” (Pérez y Uribe, 2016, p. 541). Es por ello que la Economía Solidaria, al enfocarse en las dinámicas de interés colectivo, cooperación, gratuidad y confianza, puede decirse que tiene implícita la tarea de realizar una lectura amplia del territorio, donde es posible encontrar tanto procesos conflictivos (tensiones) como acciones colectivas ${ }^{1}$.

La Economía Solidaria y su enfoque territorial brindan así fundamentos para la construcción de paz en entornos locales (González, et. al.,

1 Un referente para la comprensión de las diferentes concepciones de territorio según las comunidades étnicas en Colombia son los trabajos de la Comisión Nacional de Reparación y Reconciliación (CNRR, 2009) y de Pino y Sánchez (2016). 
2013), de aquí que se entienda la razón de ser del acuerdo firmado en Colombia entre el Gobierno y el grupo insurgente Fuerzas Armadas Revolucionarias de Colombia (FARC), en el cual se revaloriza la perspectiva solidaria y la cooperativa como ejes fundamentales para la reconstitución del tejido comunitario destruido por la guerra, sobre todo en las zonas rurales dispersas, y su interés por abordar procesos de intervención para la renovación del territorio.

Ahora bien, estudiar de manera distinta el conflicto en contextos de ruralidad en países como Colombia trae consigo, no solo la necesidad de una postura epistemológica alternativa al paradigma vigente en disciplinas como la Economía, sino también la de aplicar una aproximación metodológica distinta; en consecuencia, a continuación se esboza la propuesta metodológica triangulada y su respectiva aplicación en dos municipios en el departamento del Cauca.

\section{Metodología}

Como se mencionara antes, el pensamiento económico solidario requiere un estudio de territorio: es por ello que este documento plantea la necesidad de un enfoque social triangulado, en tanto herramienta que integra teoría, elementos cuantitativos-cualitativos y la visión integral de los sujetos de investigación y los(as) investigadores(as), distanciándose de una visión estrictamente positivista propia del análisis económico ortodoxo, según la cual es válido sólo aquello demostrable a partir de evidencias o pruebas (datos), mientras que todo aquello que no sea sujeto de medición rara vez puede ser considerado científico. Contrario a ello, en la triangulación "no se trata de complementar nuestra visión de la realidad con dos miradas [cualitativa y cuantitativa], sino de utilizar ambas orientaciones para el reconocimiento de un mismo e idéntico aspecto de la realidad social” (Triviño y Sanhueza, 2005, p. 20-21).

Según Denzin (1970), entre las características de la triangulación metodológica están la de integrar los análisis de diferentes espacios, tiempos y sujetos; el hecho de ser realizada por varios investigadores; el emplear 
más de una teoría o conceptualización; y el usar más de un método o técnica de recolección de datos. En estos términos, la triangulación permite ampliar los entramados teóricos convencionales para analizar el conflicto, garantizando el desarrollo de una investigación integral y pertinente para las zonas rurales, que aporte con elementos empíricos a la construcción de paz.

Para el caso particular abordado en este documento, la metodología triangulada se aplica entre organizaciones rurales de los municipios de El Tambo y Patía, en el departamento del Cauca (suroccidente de Colombia), con quienes se trabajaron cuatro momentos de investigación (ver Tabla 1 en anexos): un primer espacio para la reflexión y consolidación de las nociones comunitarias de Economía Solidaria y territorio (Nates, 2010); un segundo encuentro para desarrollar una herramienta participativa para la reconstrucción de la historia de cada municipio (Antequera, 2013; Centro Nacional de Memoria Histórica y University of British Columbia, 2013); un tercer taller, en el cual se aplica un instrumento hibrido de caracterización organizativa integral desde los factores solidarios, el cual incorporó los ejes de caracterización convencionales en el sector solidario y social (de parte de la Unidad Administrativa Especial de Organizaciones Solidarias, UAEOS, del Ministerio del Trabajo de Colombia, 2016), con categorías extraídas de la literatura, como es el "Factor C"; y finalmente se encuentra un compartir comunitario, convocado para la construcción de agendas temáticas e ideas de proyectos solidarios en escenarios de posconflicto.

Todos estos elementos terminan por trascender el ejercicio académico, para llegar a ser aportes a las comunidades locales acompañadas (Meredith y Quiroz, 2015), dada su capacitación en Economía Solidaria y en enfoque territorial, la generación de conciencia acerca del rescate de la memoria colectiva sobre sus territorios, el autodiagnóstico de la estructura organizacional y sus factores de mejora, y por último, la construcción comunitaria de líneas de acción futura para el sector rural. 


\section{Resultados y discusión}

El Cauca, un territorio altamente golpeado por el conflicto, contiene quizá el mayor número de municipios con graves secuelas del accionar violento en el pasado reciente. Este es el caso de los municipios de El Tambo y Patía ${ }^{2}$, en la meseta y zona sur del departamento, respectivamente, en donde se llevó a cabo la aproximación metodológica antes citada. El primer encuentro se desarrolló en las zonas rurales con asociaciones víctimas del conflicto armado y demás organizaciones, por sectores económicos destacados ${ }^{3}$, a fin de sensibilizar a la población rural de la importancia del Economía Solidaria en el Posconflicto ${ }^{4}$, y al mismo tiempo, construir con los participantes conceptos claves para la consolidación de una cultura de paz.

Las apreciaciones realizadas por la comunidad (ver Tablas 2 y 3 en anexos) responden al sentir popular sobre Economía Solidaria y territorio, y de ellas se puede resaltar la importancia de implementar perspectivas económicas diferentes, que puedan fortalecer la cultura de paz, fundamentadas en la asociatividad y el trabajo colectivo, donde su eje principal

2 Tomando en consideración la complejidad de abarcar los 42 municipios del departamento del Cauca, se propone un estudio enfocado en dos municipios, El Tambo y Patía, debido a que el Informe de hechos victimizantes de la Unidad de Atención y Reparación Integral a las Víctimas (UARIV, 2012) identifica estas zonas como dos de los principales focos de hechos en reclutamiento ilegal de menores y otras acciones de vulnerabilidad, selección que se ratifica al analizar las cifras de víctimas de minas antipersonal (MAP) y municiones usadas sin explosionar (MUSE), de hechos violentos, víctimas por atentados, número de secuestros, desplazamiento y tráfico de estupefacientes.

3 La legislación colombiana reconoce dos categorías para las organizaciones del denominado tercer sector: "Organizaciones de Economía Solidaria” y "Organizaciones Solidarias de Desarrollo", y dentro de estas últimas clasifica las asociaciones, corporaciones, fundaciones, grupos de voluntariado y organismos comunales. Para los dos estudios municipales de caso aquí presentados se contó solo con la participación de organizaciones del segundo grupo, principalmente asociaciones y fundaciones.

4 En el entendido que el conflicto, como un atributo inherente a las sociedades, no termina con la firma de un acuerdo, esto ha llevado a algunos sectores sociales a denominarle Posacuerdo. No obstante, aquí se conserva el uso del término como se le ha dado en el contexto político y económico del país. 
sean las personas y se promulgue la cooperación en los territorios (incluyendo los rurales y urbanos). Estos constructos refuerzan la idea de los teóricos acerca de la Economía Solidaria como una apuesta diferente de vivir, basada en un valor primordial como es la cooperación. Sin embargo, frente al concepto de territorio se observa que la gran mayoría lo asocia aún a una mirada geográfica, como un lugar, sitio o tierra, dejando de lado otras dimensiones intangibles que hacen parte del territorio, como son la cultura, las relaciones sociales y el vínculo con la naturaleza, entre otras. Por otro lado, se pudo identificar que existe poco conocimiento sobre el acuerdo de paz del Gobierno de Colombia y las FARC, pero al mismo tiempo una sensación de incertidumbre frente a su sostenibilidad en ambos municipios, sustentado esto en las vivencias históricas del conflicto armado en el orden local.

El segundo encuentro con las comunidades locales mencionadas en el Cauca se realizó con el fin de analizar, desde la perspectiva de los actores, la historia de sus territorios, utilizando la herramienta gráfica denominada "Diagrama histórico de los territorios". Su importancia radicó en promover la participación activa de los individuos en la investigación, con una óptica claramente de "Abajo-hacia-Arriba”, es decir, donde los problemas / soluciones (pasadas y futuras) son definidos y analizados por los propios afectados. En otras palabras, con este segundo momento se buscó dotar de mayor contenido social el ejercicio de construcción de conocimiento con las organizaciones, alrededor de su inter-dependencia con el territorio, y la necesidad de repensar los proyectos, con la comunidad y para la comunidad. El diagrama en mención se constituye de un eje vertical, que propone nueve variables (población, recursos naturales, confianza y solidaridad, producción, comercialización, conflicto, trabajo, proyectos, e instituciones), y de un eje horizontal que representa el tiempo, dividido en tres décadas (19801990; 1990-2000; 2000-2010), para finalmente realizar un cotejo con la actualidad (año 2017). 
Una aproximación a territorios en situación de conflicto armado: perspectivas desde la Economía Solidaria con un enfoque triangulado en dos municipios del Cauca (Colombia)

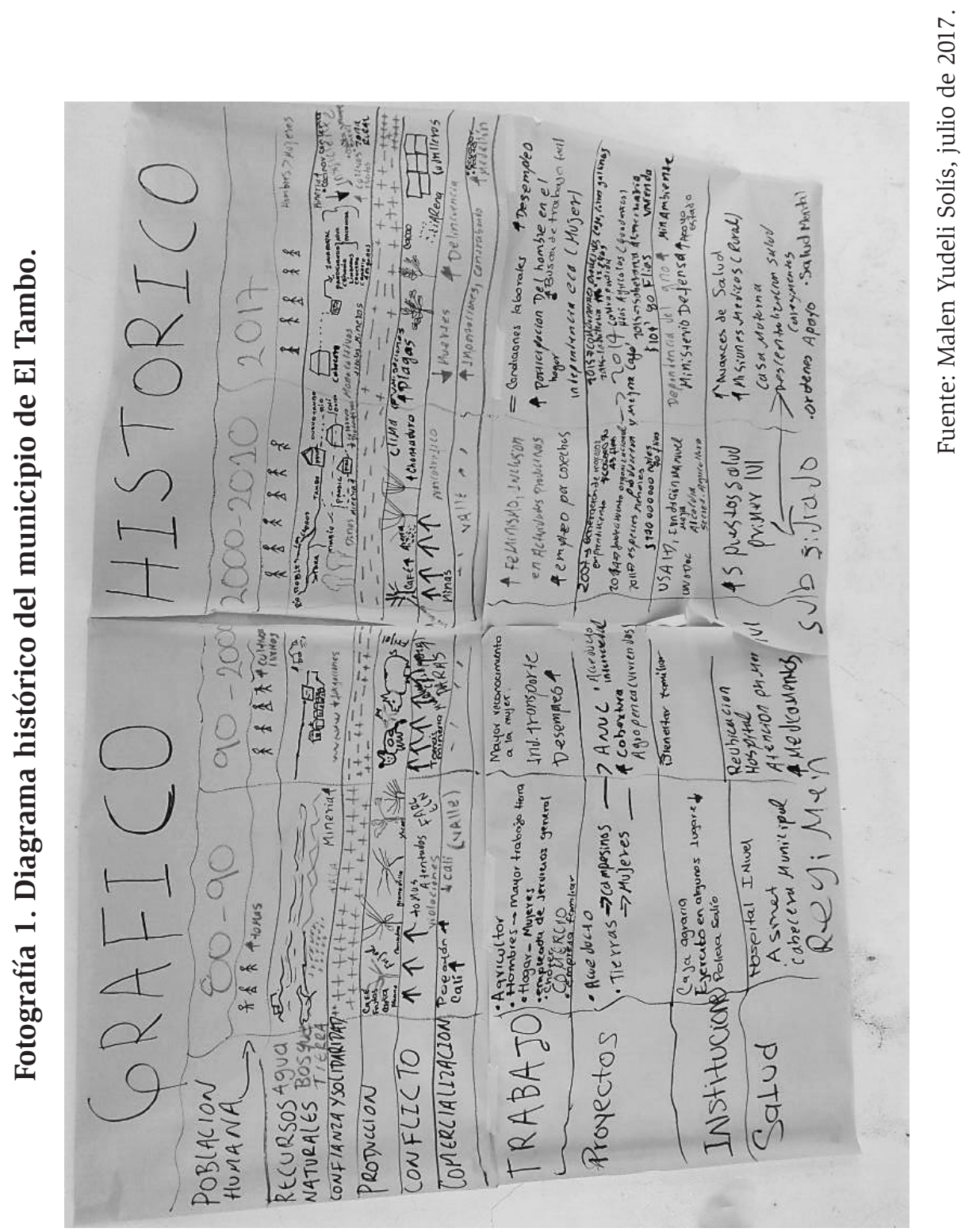


La foto 1 evidencia la construcción comunitaria del diagrama histórico del municipio de El Tambo, en la cual se plasman los aportes por medio de relatos de los(as) participantes, alrededor de las variables propuestas. Según la percepción de los actores, para el momento actual la población tambeña ha aumentado, por la llegada de nuevos actores al territorio; las acciones de asociatividad basadas en la solidaridad y confianza se han plasmado a través del aumento de asociaciones de base y trabajo comunitario (hecho que confirma lo mostrado en estadísticas oficiales), al paralelo que las muertes a causa de la guerra disminuyen, si bien los hechos de delincuencia común no han cedido; en materia ambiental, argumentan los lugareños, actualmente los recursos naturales son escasos (en especial la disponibilidad de agua), existe todavía una gran presencia de minería ilegal y, si bien el acceso al mercado es mayor que en el pasado, las dificultades para la consolidación de un comercio justo aún son evidentes.

Por su parte, la información obtenida en el municipio de Patía, como ilustra la foto 2 , indica una transformación de las distintas variables del territorio, siendo mayormente preocupante para la población local la delincuencia y el micro-tráfico, y el deterioro de los recursos naturales, los cuales disminuyen a medida que la población aumenta. En términos generales, en ambos territorios el conflicto social y armado causó la salida de familias, vía desplazamiento forzado, pero también llegada de otros actores, debido a las actividades ilegales; la inseguridad todavía sigue rompiendo los lazos sociales en los territorios, pero, en reacción a este fenómeno, el incremento de instituciones y la autogestión de las comunidades han hecho posible el establecimiento de iniciativas solidarias en sus corregimientos, que contribuyen a mejorar las condiciones de vida locales. 


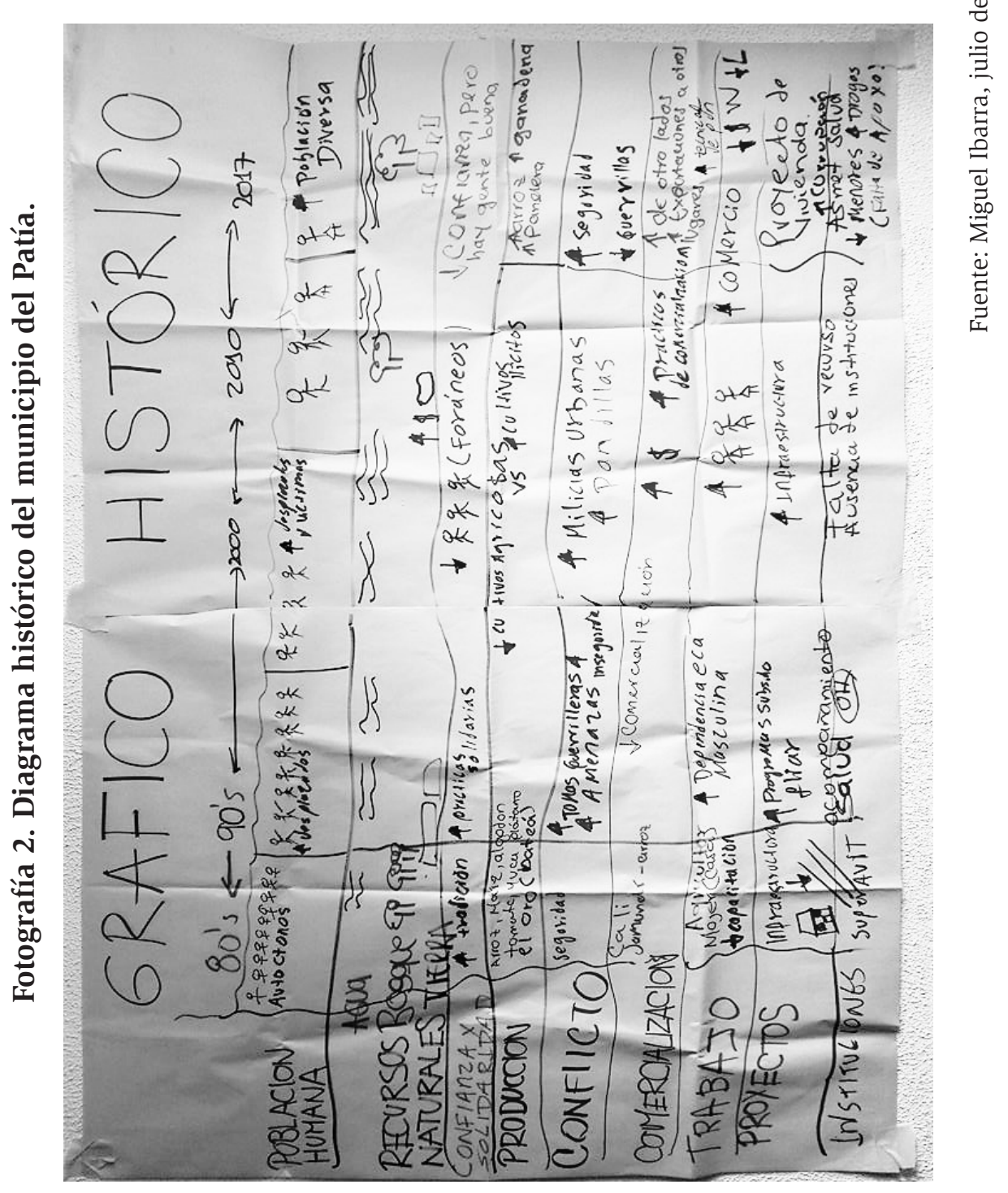


Posteriormente, un tercer encuentro fue llevado a cabo para realizar una caracterización de organizaciones con enfoque solidario en los municipios de El Tambo y Patía, con la participación de un reducido pero significativo número (23 y 17 organizaciones, respectivamente). En esta jornada se posibilitó la aplicación de un instrumento híbrido, con el fin de establecer un análisis organizativo integral, que permitió reunir información valiosa del tejido asociativo en los municipios de El Tambo y Patía (ver Figura 1 en anexos), en frentes frecuentemente citados por los organismos del Estado, pero difícilmente validados y publicados, a saber: que las comunidades rurales, y las organizaciones en ellas constituidas, presentan aun dificultades para la generación de valor agregado sobre sus productos, no tienen forma de aprovechar las posibilidades del internet para comercializar bienes y servicios, no cuentan con portafolio de servicios y de indicadores de satisfacción, tanto para clientes como asociados, si bien tienen por fortaleza el amplio "Factor C” (cooperación interna, compañerismo, colaboración), clave dentro de la gestión de los procesos organizativos; se destacan aquí también la capacidad de resolver conflictos, el respeto al medio ambiente, los procesos y las actividades inclusivas, de participación, e intercambio de conocimientos y saberes entre las diferentes generaciones.

No obstante, hay aspectos como los jurídicos, y de gestión, como el enfoque a los grupos de interés (stakeholders), donde las dificultades son notorias: falta de actualización de estatutos, de renovación ante Cámara de Comercio, obtención del número de identificación tributaria (NIT), realización periódica de asambleas, débil instauración de actas y reglamentos internos para sus respectivos órganos de administración y control, insuficiente presupuesto anual, bajo control en el pago de aportes y distribución de excedentes (cuando se logran), débil financiamiento. Como generalidad, la mayoría de organizaciones solidarias de desarrollo participantes afirman haber tenido dificultades en el desarrollo de sus actividades económicas debido al conflicto armado y coinciden en no tener capacitación para formulación de proyectos; a pesar de esto, conservan sus fundamentos solidarios y territoriales, que deben fortalecerse, 
ya que, por ejemplo, han olvidado aplicar acciones de soberanía alimentaria, agroecológicas, procesos interculturales o educativos.

El último encuentro con las organizaciones rurales de El Tambo y Patía se llevó a cabo teniendo en cuenta los acuerdos firmados en La Habana entre las FARC y el Gobierno nacional, y el hecho de que las organizaciones deben estar preparadas para la cofinanciación de sus proyectos de Economía Solidaria. Así, en primer lugar se identificaron las líneas estratégicas: para el caso de las organizaciones del municipio del El Tambo, café, frutas y verduras, ganado, cerdos, reforestación, plátano, turismo, productos agrícolas, pollos y chontaduro, las cuales responden a líneas tradicionales de producción, que dada la experiencia e infraestructura existente puede generar ingresos económicos a los asociados, y en algunos casos para sus familias y/o comunidad, aunque se referencian también iniciativas más allá de lo productivo, como son la reforestación de la micro cuenca del rio Piagua (zona centro), para que beneficie al municipio, y la transformación de materiales reciclables para obtener artesanías, para que beneficie a las víctimas del conflicto armado. Así mismo, en el municipio del Patía, las ideas de proyectos económicos de las organizaciones giran alrededor de las frutas, avicultura, productos agrícolas, cacao, economía ambiental y social, apicultura, porcicultura, ganadería, manufactura; en esta zona, aunque existe tendencia hacia la formulación de proyectos productivos en un contexto de posconflicto, a diferencia de El Tambo, se tienen más ideas de proyectos sociales y ambientales que beneficien a la región y que buscan mejorar las condiciones de convivencia, la promoción de la cultura ecológica, una mayor atracción turística y de recreación, y en general el beneficio a las personas en situación de vulnerabilidad como niños, discapacitados y víctimas del conflicto armado.

\section{Conclusiones}

Desde la visión económica ortodoxa se ha omitido el conflicto como una categoría analítica central, ya que se asume que los individuos toman decisiones racionales, siendo incluso lógico el auge de las 
actividades ilegales, debido a que estas generan mayor retribución económica que las legales; en efecto, las Ciencias Económicas actualmente no discuten de manera suficiente aspectos tan fundamentales como las pugnas sociales derivadas de la desigualdad económica, y más allá, las acciones institucionales y de asociatividad para fines comunes, como sí lo plantea la Economía Solidaria, enfoque teórico y aplicado centrado en las personas y los territorios, donde se estructuran lazos sociales. En consecuencia, este artículo deja planteada, como primera medida, la necesidad de utilizar este marco teórico para futuras investigaciones en zonas en conflicto.

Adicionalmente, el documento logra mostrar la pertinencia que tiene el enfoque metodológico triangulado, donde se integran los planteamientos teóricos (en este caso desde la Economía Solidaria y el territorio) con las voces, acciones y propuestas comunitarias, todo lo cual conduce a una mejor entendimiento de los contextos locales; en particular, se muestra la importancia de un reconocimiento comunitario de las transformaciones temporales en los territorios (análisis cualitativo histórico), para comprender desde esta visión las características que deben tener los nuevos proyectos colectivos a futuro, así como la caracterización integral (cualitativa y cuantitativa) de las organizaciones que han surgido en los territorios, fruto de lo cual es posible sugerir mejoras de gestión, entre ellas, en las características de sus proyectos solidarios y en la generación de beneficios sociales.

En términos generales, se puede concluir también la necesidad de agendas temáticas y proyectos que fortalezcan las apuestas productivas históricas en el sector rural, pero que involucren mayormente una perspectiva social, solidaria y territorial en beneficio de la comunidad, de los niños y de las víctimas del conflicto armado, que fortalezca los lazos de confianza y convivencia, y promueva la paz en el marco de un contexto del posconflicto. En este sentido es fundamental que las organizaciones interactúen con otros actores institucionales, tales como las alcaldías municipales, los Ministerios del Gobierno, la Unidad 
administrativa especial de organizaciones solidarias (UAEOS), el Sistema General de Regalías y Cooperación internacional, pero sin descuidar la finalidad solidaria de sus proyectos y la autonomía que les asiste como constructores(as) del territorio.

En este último aspecto, paralelamente al fortalecimiento de los factores de gestión de las organizaciones sociales y solidarias de los territorios rurales, como los aquí abordados para el departamento del Cauca, se requiere a futuro el estudio de la legislación que pueda representar oportunidades para las comunidades. Se refiere esto a los decretos y leyes de implementación de la llamada "Reforma rural integral” para el acceso a tierras, la formalización y el ordenamiento de las propiedades, la sustitución de cultivos de uso ilícito, los beneficios por implementación de servicios ambientales, etc. Todo ello en un marco de autonomía respecto a las instituciones del Gobierno vigente, haciendo prevalecer los derechos de los pueblos a la economía propia y a la vida digna, materializados en figuras como las zonas de reserva campesinas, los territorios interculturales, los territorios campesinos agroalimentarios, etc.

\section{Referencias}

Agudelo, María; Patiño, Beatriz y Ramos, Michael (2010). Conceptualización del factor C: antecedentes y perspectivas. Revista Ingeniería Solidaria, vol. 6, 95-101.

Antequera, José Darío (2013). Memoria histórica como relato emblemático (Tesis de Maestría). Bogotá: Pontificia Universidad Javeriana.

Becker, Gary Stanley (1968). Crime and Punishment: An Economic Approach. The Journal of Political Economy, 76:2, 169-217.

Bowles, Samuel (2010). Microeconomía. Comportamiento instituciones y evolución. Recuperado de: https://bowlesmicroeconomia.uniandes.edu.co/ capitulos/Microeconomia_Bowles_Completo.pdf

Centro Nacional de Memoria Histórica y University of British Columbia (2013). Recordar y narrar el conflicto. Recuperado de: http://www.centrodememoriahistorica.gov.co/descargas/informes2009/recordar-narrar-el-conflicto.pdf 
CNRR (2009). Territorio y despojo. En: El despojo de tierras y territorios aproximación conceptual. 77-97. Recuperado de: http://www.centrodememoriahistorica.gov.co/descargas/informes2010/tierra_conflicto/despojo_tierras_baja.pdf

Coraggio, José Luis (2011). Economía social y solidaria. El trabajo antes que el capital. Quito: Ediciones Abya Yala. Recuperado de: http://www.coraggioeconomia.org/jlc/archivos \%20para \% 20descargar/economiasocial.pdf

Denzin, Norman (1970). The Research Act. Chicago: Aldine.

Gonzáles, Jorge (1995). El conflicto entre el interés privado y el interés colectivo. Una invitación a leer a Veblen. Revista de Ciencias Administrativas y Sociales. Recuperado de: http://www.bdigital.unal.edu.co/22513/1/1913862717-1-PB.pdf

Gonzáles, Camilo et al. (2013). Conversaciones en La Habana: claves para construir la Paz. Bogotá: Editorial Oveja Negra.

Haavelmo, Trygve (1944). The probability approach in econometrics. Cowles Foundation, Paper no. 4. USA: Cowles Foundation for Research in Economics, Yale University. Recuperado de: http://fitelson.org/woodward/ haavelmo.pdf

Iglesias, Emilia (2013). La mediación como método para la resolución de conflictos. Revista de trabajo social de Murcia, núm. 18. Recuperado de: https://dialnet.unirioja.es/descarga/articulo/4549233.pdf

Jácome, Hugo (2016). Avances y desafíos de la economía popular y solidaria en el Ecuador. En Jácome, Hugo et al. Economía solidaria. Historias y prácticas de su fortalecimiento, 19-34. Quito: Superintendencia de Economía Popular y Solidaria, Serie estudios sobre la Economía Popular y Solidaria. Recuperado de: http:// www.seps.gob.ec/documents/20181/26626/Libro-Economi\%CC\% 81a \% 20Solidaria. \% 20Historias \% 20y \% 20pra \% CC \% 81cticas \% 20de\%20su \% 20fortalecimiento2016\%20.pdf/ff618ad6-b4b6-497b-abe1-24b0ab16605e

Manzano, Bernardo (2005). Territorio, teoría y política. Publicación del Programa de Post-Graduación en Geografía de la Universidad Estadual Paulista - UNESP. Recuperado de: https://problemasrurales.files.wordpress. com/2008/12/territorio-teoria-y-politica-bernardo.pdf 
Marx, Karl (2004). El Capital (El proceso de producción del Capital, tomo 1, vol.1). México, DF: Siglo XXI, Colección Biblioteca del Pensamiento Socialista.

Meredith, Margareth y Quiroz Niño, Catalina (coords.) (2015). Consolidando estudios y prácticas de la economía social y solidaria. York: Consorcio York St John-Erasmus sobre Economía Social y Solidaria. Recuperado de: http:// base.socioeco.org/docs/cap_5_es_v1.6_31.08.2016.pdf https://www.yorksj. ac.uk/socialeconomy

Moncayo, Edgar (2001). Evolución de los paradigmas y modelos interpretativos de desarrollo territorial. Revista CEPAL, 13, 5-47.

(2003). Nuevas teorías y enfoques conceptuales sobre el desarrollo regional: ¿hacia un nuevo paradigma? Revista de economía institucional, $5,32-65$.

Nates, Beatriz. (2010). Soportes teóricos y etnográficos sobre conceptos de territorio. Revista Co-herencia, 8, 14, 209-229.

Pastore, Rodolfo (2006). Diversidad de trayectorias, aproximación conceptual y pluralidad de proyectos de la economía social. Documento 54. Argentina: Universidad de Buenos Aires. Facultad de Ciencias Económicas. Centro de Estudios de Sociología, 1-17. Recuperado de: http://bibliotecadigital.econ. uba.ar $/ ? \mathrm{a}=\mathrm{d} \& \mathrm{c}=$ docin\&d = docin_cesot_054

Pérez, Edelmira (2001). Hacia una nueva visión de lo rural. Buenos Aires: CLACSO. Recuperado de: http://bibliotecavirtual.clacso.org.ar/clacso/gt/20100929011414/2perez. pdf

Pérez, Pastor y Uribe, Victor (2016). Reflexiones para conceptualizar territorio solidario. Revista de ciencias sociales El Ágora USB, vol.16, núm. 2, 533546. Recuperado de: DOI: http://dx.doi.org/10.21500/16578031.2446

Pino, Yenny y Sánchez, David (2016). Pedagogía y territorio. La experiencia de la Escuela de Derechos Humanos y ciudadanía en el Bajo Cauca Antioqueño. Revista de ciencias sociales El Ágora USB, 7(1), 35-57.

Prebisch, Raúl (1983). Centro y periferia en el origen y maduración de la crisis. Pensamiento Iberoamericano, (3), 27-40. 
Primavera, Heloisa (2011). Moneda social y democracia: manual para comprender y hacer ( $2^{a}$ versión). Recuperado de: http://www.economiasolidaria.org/documentos/moneda_social_y_democracia_manual_para_ comprender_y_hacer_2v

Razeto, Luis (1995). Los caminos de la economía de la solidaridad. Recuperado de: http://lacoperacha.org.mx/documentos/coperacha-economia-solidariarazeto.pdf

(2010). ¿Qué es economía solidaria? Papeles de relaciones eco sociales y cambio global, 110, 47-52.

Rawls, John (2006). Teoría de la justicia. México: Fondo de Cultura Económica.

Salgado, Carlos (2005). Los campesinos imaginados. Cuadernos tierra y justicia, 6, 8-41.

Schejiman, Alexander (1980). Economía campesina: lógica interna, articulación y persistencia. Revista CEPAL, 11, 121-140.

Triviño, Zaider y Sanhueza, Olivia (2005). Paradigmas de investigación en enfermería. Ciencia Enfermería, vol. 11, 1, 17-24.

Unidad Administrativa Especial de Organizaciones Solidarias (UAEOS), Ministerio del Trabajo (2016). Informe de Gestión y Resultados 2016. Revista Organizaciones Solidarias, edición núm. 22. Recuperado de: http://www. orgsolidarias.gov.co/sites/default/files/archivos/RENDICION \% 20DE \% 20 CUENTAS\% 202017.pdf

Unidad para la atención y reparación de victimas (UARIV) (2012). Cauca: Informe Departamental de Hechos Victimizantes a 2012. Documento técnico. Disponible en: http://rni.unidadvictimas.gov.co/sites/default/files/Documentos/Cauca.pdf Consultado el 19-10-16

Veblen, Thorstein (2000). Teoría de la clase ociosa. Ediciones elaleph.com. (Cap. XII. Supervivencias del interés no valorativo, 350-381). Recuperado de: https://es.scribd.com/doc/7125234/Thorstein-Veblen-Teoria-de-LaClase-Ociosa 